\title{
Existence and Continuity of Solutions to a Class of Pseudodifferential Equations over $p$-Adic Field
}

\author{
Bo Wu \\ Department of Applied Mathematics, Nanjing University of Finance \& Economics, Nanjing 210023, China \\ Correspondence should be addressed to Wu Bo; bowu8800@gmail.com
}

Received 12 April 2013; Accepted 19 May 2013

Academic Editor: Guo-Cheng Wu

Copyright (C) 2013 Bo Wu. This is an open access article distributed under the Creative Commons Attribution License, which permits unrestricted use, distribution, and reproduction in any medium, provided the original work is properly cited.

We study the pseudodifferential operator $T^{\alpha}$ and the pseudodifferential equations of type $T^{\alpha} u+u=\delta_{x_{k}}$ over $p$-adic field $\mathbb{Q}_{p}$, where $\delta_{x_{k}}$ is the Dirac delta function. We discuss the existence and uniqueness of solutions to the equations. Furthermore, we give conditions for the continuity of the solutions $u_{k}$ when $u$ belongs to the space $L_{2}\left(\mathbb{Q}_{p}\right)$.

\section{Introduction}

In recent years $p$-adic analysis has received a lot of attention due to its applications in mathematical physics; see, for example, $[1-13]$ and references therein.

A good example of the applications is the pseudodifferential equations on the field $\mathbb{Q}_{p}$. In 60s of the last century, Gibbs first gives logic derivative on dyadic field. Since then, Vladimirov et al. [13] extended logic derivative to $p$-adic field and made the certain correction of the original Gibbs definition. This kind of derivative referred to Vladimirov pseudodifferential operator. Kuzhel and Albeverio et al. used Vladimirov operator to study pseudodifferential equations; and see, for example, [14-16]. However, the Vladimirov pseudodifferential operator, as a kind of operation, is not closed in the test function space $S\left(\mathbb{Q}_{p}\right)$. This makes the definition of Vladimirov operator difficult to be applied to distribution space $S^{\prime}\left(\mathbb{Q}_{p}\right)$. In 1992, Su [17] defined derivative and integral operator $T^{\alpha}$ on locally compact Vilenkin groups $G$. The space of test functions space $S(G)$ and its distribution $S^{\prime}(G)$ with the operation of $T^{\alpha}$ is closed. $T^{\alpha}$ is defined as a pseudodifferential operator with the symbol $\langle\xi\rangle^{\alpha}$

$$
T^{\alpha} \varphi=\left(\langle\xi\rangle^{\alpha} \varphi^{\wedge}(\xi)\right)^{\vee},
$$

where $\langle\xi\rangle=\max \{1,|\xi|\}, \alpha \in \mathbb{R}$. The new definition plays a role in promoting the development of theory for $p$-adic analysis.
In this paper, we study a class of pseudodifferential equations of type

$$
T^{\alpha} u+u=\delta_{x_{k}}, \quad u \in L_{2}\left(\mathbb{Q}_{p}\right), x_{k} \in \mathbb{Q}_{p},
$$

where $T^{\alpha}$ is pseudodifferential operator defined by Su and $\mathbb{Q}_{p}$ is the field of $p$-adic numbers.

Our aim is to show that (2) has a unique solution $u_{k}$ belonging to $L_{2}\left(\mathbb{Q}_{p}\right)$ if $\alpha>1 / 2$ and has no solutions if $\alpha \leq$ $1 / 2$.

Moreover, we give the condition for the continuity of the solutions $u_{k}$ with the index $\alpha$.

\section{Preliminaries}

We use the notations indicated in Taiblesons book [18]. Let $p \geq 2$ be a prime number. The field $\mathbb{Q}_{p}$ of $p$-adic numbers is a topologically complete space of rational numbers $\mathbb{Q}$ with respect to $p$-adic norm $|\cdot|_{p}$ (non-Archimedean norm), which is defined as follows:

if $x=0$, then $|0|_{p}=0$;

if $x \neq 0$ is an arbitrary rational number, we define $|x|_{p}=p^{-r}$. And $x$ can be represented as $x=p^{r}(m / n)$, where $r=\operatorname{order}_{p} x \in \mathbb{Z}$ and integers $m$ and $n$ are relatively primes and not divisible by $p$.

The $p$-adic norm $|\cdot|_{p}$ satisfies the strong triangle inequality $|x+y|_{p} \leq \max \left(|x|_{p},|y|_{p}\right)$. 
Any $p$-adic number $x \neq 0$ in the topologically complete space $\mathbb{Q}_{p}$ can be presented as series uniquely:

$$
\begin{gathered}
x=\sum_{i=m}^{+\infty} x_{i} p^{i}, \\
0 \leq x_{i} \leq p-1, \quad i=m, m+1, \ldots, \quad m \in \mathbb{Z}, \quad x_{m} \neq 0 .
\end{gathered}
$$

Define the bitwise operation of addition and multiplication of $x$ in $\mathbb{Q}_{p}$ (either from left to right carry, or not carry); then $\mathbb{Q}_{p}$ is a locally compact, totally disconnected, and complete topological field.

Denote by $B_{0}=\left\{x \in \mathbb{Q}_{p}:|x|_{p} \leq 1\right\}$ the ring of integers in $\mathbb{Q}_{p}$ which is the subring of $\mathbb{Q}_{p} . B_{0}$ is the compact subring and compact subspace of locally compact field $\mathbb{Q}_{p}$. With the addition operation of $p$-adic field $\mathbb{Q}_{p}$, there exists the Haar measure $d x$ on $\mathbb{Q}_{p}$ such that $\int_{B_{0}} d x=1$. Denote by

$$
\begin{aligned}
& B_{r}=\left\{x \in \mathbb{Q}_{p}:|x|_{p} \leq p^{r}\right\}, \\
& S_{r}=\left\{x \in \mathbb{Q}_{p}:|x|_{p}=p^{r}\right\},
\end{aligned}
$$

respectively, the ball and the sphere of radius $p^{r}$ with the center at $0 \in \mathbb{Q}_{p}$. Obviously, $\int_{B_{r}} d x=p^{r}, \int_{S_{r}} d x=p^{r}\left(1-p^{-1}\right)$.

A complex-valued function $\varphi: \mathbb{Q}_{p} \rightarrow \mathbb{C}$ defined on $\mathbb{Q}_{p}$ is called locally constant if for any $x \in \mathbb{Q}_{p}$ there exists an integer $l(x) \in \mathbb{Z}$ satisfying

$$
\varphi\left(x+x^{\prime}\right)=\varphi(x), \quad \forall x^{\prime} \in B_{l(x)} .
$$

Denote by $\varepsilon=\varepsilon\left(\mathbb{Q}_{p}\right)$ the linear space of all the locally constant functions. $S=S\left(\mathbb{Q}_{p}\right)$ is defined as the linear space of all locally constant functions with compact support in $\mathbb{Q}_{p}$.

The convergence of the point in $\varepsilon$ according to the following definition: $\varphi_{k} \stackrel{\varepsilon}{\rightarrow} 0, k \rightarrow \infty$ if and only if for any compact subset $E \subset \mathbb{Q}_{p}, \varphi_{k} \rightarrow 0, k \rightarrow \infty$ holds uniformly on $E$. The convergence in $S$ has the following meaning: $\varphi_{k} \stackrel{S}{\rightarrow} 0$, $k \rightarrow \infty$ if and only if there exists the indices $l$ and $N$ which do not depend on $k$, such that the functions $\left\{\varphi_{k}\right\}$ with supports in the ball $B_{N}$ and with constant on the coset of $B_{l}$, $\varphi_{k} \rightarrow 0, k \rightarrow \infty$ hold uniformly in $B_{N}$. Then, $\varepsilon$ and $S$ are complete topological linear spaces. Also denote by $S$ the test function space. formula

The Fourier transform of $\varphi \in S\left(\mathbb{Q}_{p}\right)$ is defined by the

$$
\varphi^{\wedge}(\xi)=\int_{\mathbb{Q}_{p}} \varphi(x) \overline{\chi_{p}}(\xi x) d x, \quad \xi \in \mathbb{Q}_{p},
$$

and inverse Fourier transform $\varphi^{\vee}$ by

$$
\varphi^{\vee}(x)=\int_{\mathbb{Q}_{p}} \varphi(\xi) \chi_{p}(\xi x) d \xi, \quad x \in \mathbb{Q}_{p},
$$

where $\chi_{p}(x)=e^{2 \pi i\{x\}}$ is an additive character of the field $\mathbb{Q}_{p}$, with value 1 in $B_{0}$, and $\{x\}=x_{-1} p^{-1}+x_{-2} p^{-2}+\cdots+x_{m} p^{m}$. The
Fourier transform and inverse Fourier transform map $S\left(\mathbb{Q}_{p}\right)$ onto $S\left(\mathbb{Q}_{p}\right)$.

Denote by $S^{\prime}=S^{\prime}\left(\mathbb{Q}_{p}\right)$ the distribution space of test function space $S . S^{\prime}$ is a complete topological linear space under the dual topology. The convergence in $S^{\prime}$ according to the following definition: $f_{k} \stackrel{s^{\prime}}{\rightarrow} 0, k \rightarrow \infty$ if and only if $\left\langle f_{k}, \varphi\right\rangle \rightarrow 0, k \rightarrow \infty$ holds for any $\varphi \in S$.

It follows from the definition of $S\left(\mathbb{Q}_{p}\right)$ that any test function $\varphi \in S\left(\mathbb{Q}_{p}\right)$ is continuous on $\mathbb{Q}_{p}$. This means the Dirac delta function $\left\langle\delta_{x}, \varphi\right\rangle=\varphi(x)$ is well posed for any point $x \in \mathbb{Q}_{p}$.

Denote by $L_{2}\left(\mathbb{Q}_{p}\right)$ the set of the measurable functions $f$ on $\mathbb{Q}_{p}$ with the condition $\int_{\mathbb{Q}_{p}}|f(x)|^{2} d x<\infty$. And set $L_{2}\left(\mathbb{Q}_{p}\right)$ is a Hilbert space satisfying the scalar product $(f, g)_{L_{2}\left(\mathbb{Q}_{p}\right)}=$ $\int_{\mathbb{Q}_{p}} f(x) \overline{g(x)} d x$.

Let $E$ be compact set, and $1_{E}$ is the indicative function of set $E$. Then there exist standard sequences $\Delta_{k}(x)$ of 1 satisfying $\Delta_{k}(x)=1_{B_{k}}, k \in \mathbb{Z}, x \in \mathbb{Q}_{p}$.

\section{Pseudodifferential Operator $T^{\alpha}$}

In 1992, Su [17] has given definitions of the derivative for the $p$-adic local fields $\mathbb{Q}_{p}$, including derivatives of the fractional orders and real orders.

Let $\xi \in \mathbb{Q}_{p}$ and $\langle\xi\rangle=\max \{1,|\xi|\}$. Its role is played by the operator of pseudodifferential operator $T^{\alpha}(\alpha \in \mathbb{R})$ which is defined as

$$
T^{\alpha} \varphi(x)=\left(\langle\xi\rangle^{\alpha} \varphi^{\wedge}(\xi)\right)^{\vee}(x),
$$

for $\varphi \in S\left(\mathbb{Q}_{p}\right)$. It is easy to see that $T^{\alpha} \varphi \in S\left(\mathbb{Q}_{p}\right)$. With

$$
\left\langle T^{\alpha} f, \varphi\right\rangle=\left\langle f, T^{\alpha} \varphi\right\rangle, \quad f \in S^{\prime}\left(\mathbb{Q}_{p}\right), \varphi \in S\left(\mathbb{Q}_{p}\right),
$$

the defined domain of $T^{\alpha}$ can be extended to the space $S^{\prime}\left(\mathbb{Q}_{p}\right)$. Thus, we also have $T^{\alpha} f=\left(\langle\xi\rangle^{\alpha} f^{\wedge}(\xi)\right)^{\vee}$ with $f \in S^{\prime}\left(\mathbb{Q}_{p}\right)$ and $T^{\alpha} f \in S^{\prime}\left(\mathbb{Q}_{p}\right)$.

Definition 1. If $\alpha>0$, then $T^{\alpha}$ is defined as $p$-adic derivatives of the order $\alpha$ on $S^{\prime}\left(\mathbb{Q}_{p}\right)$. And if $\alpha<0$, then $T^{\alpha}$ is defined as $p$-adic integral of the order $-\alpha$ on $S^{\prime}\left(\mathbb{Q}_{p}\right)$. If $\alpha=0, T^{0} f=f$ for any $f \in S^{\prime}$, then $T^{0}$ is called the identity operator.

In what follows we consider $T^{\alpha}$ as an operator in Hilbert space $L_{2}\left(\mathbb{Q}_{p}\right)=L_{2}$. Obviously, the set of functions $D\left(T^{\alpha}\right)=$ $\left\{f \in L_{2}:\langle\xi\rangle^{\alpha} f^{\wedge} \in L_{2}\right\}$ is the domain of definition of $T^{\alpha}$ on the space $L_{2}$

$$
T^{\alpha} f=\left(\langle\xi\rangle^{\alpha} f^{\wedge}\right)^{\vee}
$$

In [19], Qiu and Su have recently studied the spectrum of $T^{\alpha}$ and constructed the set of eigenfunctions of $T^{\alpha}$ :

$$
\begin{gathered}
\psi_{N j I}(x)=p^{-N / 2} \chi\left(p^{N-1} j x\right) \Delta_{0}\left(p^{N} x-z_{I}\right), \\
N \in \mathbb{Z}, \quad I=z_{I}+B_{0} \in \frac{\mathbb{Q}_{p}}{B_{0}}, \quad j=1,2, \ldots, p-1
\end{gathered}
$$


which forms an orthonormal basis in $L_{2}\left(\mathbb{Q}_{p}\right)$ ( $p$-adic wavelet basis) such that

$$
T^{\alpha} \psi_{N j I}(x)= \begin{cases}p^{(1-N) \alpha} \psi_{N j I}(x), & N<1, \\ \psi_{N j I}(x), & N \geq 1 .\end{cases}
$$

Theorem 2. The function $f$ is continuous in $D\left(T^{\alpha}\right)$ if and only if $\alpha>1 / 2$.

Proof. Let $f \in D\left(T^{\alpha}\right)$; we expand the function $f$ under the $p$-adic wavelet basic (11) as follows:

$$
\begin{aligned}
f(x)= & \sum_{N=1}^{\infty} \sum_{j=1}^{p-1} \sum_{I}\left(f, \psi_{N j I}\right) \psi_{N j I}(x) \\
& +\sum_{N=-\infty}^{0} \sum_{j=1}^{p-1} \sum_{I}\left(f, \psi_{N j I}\right) \psi_{N j I}(x) .
\end{aligned}
$$

Evidently, $\psi_{N j I}(x)$ belong to the domain of definition of $T^{\alpha}$, and then, the functions $\psi_{N j I}(x)$ are continuous on $\mathbb{Q}_{p}$. Thus, it is sufficient to prove the continuity of $f(x)$ with verifying that the series (13) converges uniformly.

First, we note that there is at most one $z_{I}$ such that $\psi_{N j I}(x) \neq 0$ for the fixed $N$ and $x$. Indeed, if there are $z_{I_{1}}$ and $z_{I_{2}}$ satisfying $\psi_{N j I}(x) \neq 0$, then we have $\Delta_{0}\left(p^{N} x-z_{I_{i}}\right)=1$. But $\left|p^{N} x-z_{I_{1}}\right| \leq 1$ and $\left|p^{N} x-z_{I_{2}}\right| \leq 1$. With the strong triangle inequality $\left|z_{I_{1}}-z_{I_{2}}\right| \leq 1$. From the condition $I=z_{I}+B_{0} \in$ $\mathbb{Q}_{p} / B_{0}$, we can get the equality $I_{1}=I_{2}$.

Thus, the sum with the parameter $I$ consists of at most one nonzero term for fixed $N$ and $x$.

Further, following from (11) and (13), we obtain

$$
\left|\psi_{N j I}(x)\right| \leq p^{-N / 2}, \quad\left|\left(f, \psi_{N j I}\right)\right| \leq\|f\|_{L_{2}(Q)} .
$$

The relations (14) obtain the following estimate if $N \geq 1$

$$
\left|\sum_{j=1}^{p-1} \sum_{I}\left(f, \psi_{N j I}\right) \psi_{N j I}(x)\right| \leq p^{-N / 2}\|f\|_{L_{2}(Q) p}(p-1) .
$$

The estimate gives the uniform convergency of the first series in (13).

The condition $f \in D\left(T^{\alpha}\right)$ and (12) imply $\left(f, \psi_{N j I}\right)=$ $p^{(N-1)}\left(T^{\alpha} f, \psi_{N j I}\right)$ for $N<1$. For a fixed $N<1$, using CauchySchwarz equality and (14), we obtain

$$
\begin{aligned}
\left|\sum_{j=1}^{p-1} \sum_{I}\left(f, \psi_{N j I}\right) \psi_{N j I}(x)\right| \\
=\left|\sum_{j=1}^{p-1} \sum_{I} p^{(N-1)}\left(T^{\alpha} f, \psi_{N j I}\right) \psi_{N j I}(x)\right| \\
\leq\left\{\sum_{j=1}^{p-1} \sum_{I}\left|\left(T^{\alpha} f, \psi_{N j I}\right)\right|^{2}\right\}^{1 / 2} \\
\quad \times\left\{\sum_{j=1}^{p-1} \sum_{I} p^{2(N-1)}\left|\psi_{N j I}(x)\right|^{2}\right\}^{1 / 2} \\
\leq\left\|T^{\alpha} f\right\|_{L_{2}(Q)}\left\{\sum_{j=1}^{p-1} p^{-N+2(N-1)}\right\}^{1 / 2} .
\end{aligned}
$$

The estimate obtained above means the second series in (13) is uniformly convergent if $\alpha>1 / 2$. Thus, function $f$ is continuous in $D\left(T^{\alpha}\right)$ on $\mathbb{Q}_{p}$ for $\alpha>1 / 2$. Theorem 2 is proved.

Next when $\alpha \leq 1 / 2$, we will give an example in which the function belongs to $D\left(T^{\alpha}\right)$ but is not continuous on $\mathbb{Q}_{p}$.

Example 3. The function

$$
f(x)=\sum_{-\infty}^{-1} \frac{1}{|N|} p^{(N-1) / 2} \psi_{N 10}(x)
$$

belongs to $D\left(T^{\alpha}\right)$ but $f(x)$ is not continuous on $\mathbb{Q}_{p}$ for $\alpha \leq$ $1 / 2$.

It is easy to see $f \in L_{2}\left(\mathbb{Q}_{p}\right)$ and its Fourier transform is

$$
f^{\wedge}(\xi)=\sum_{-\infty}^{-1} \frac{1}{|N|} p^{(N-1) / 2} \psi_{N 10}^{\wedge}(\xi) .
$$

From (8) and (12), $\langle\xi\rangle^{\alpha} \psi_{N 10}^{\wedge}(\xi)=p^{\alpha(1-N)} \psi_{N 10}^{\wedge}(\xi)$. Thus

$$
\langle\xi\rangle^{\alpha} f^{\wedge}(\xi)=\sum_{-\infty}^{-1} \frac{1}{|N|} p^{(N-1) / 2} p^{\alpha(1-N)} \psi_{N 10}^{\wedge}(\xi) .
$$

$\langle\xi\rangle^{\alpha} f^{\wedge}(\xi) \in L_{2}\left(\mathbb{Q}_{p}\right)$ for $\alpha \leq 1 / 2$ and $\langle\xi\rangle^{\alpha} f^{\wedge}(\xi) \notin L_{2}\left(\mathbb{Q}_{p}\right)$ for $\alpha>1 / 2$ with the orthonormal basis $\left\{\psi_{N 10}\right\}_{N<0}$. So, $f(x) \in$ $D\left(T^{\alpha}\right)$ for $\alpha \leq 1 / 2$.

Next, We will show that $f(x)$ is not continuous on $\mathbb{Q}_{p}$. First, using (11), we rewrite the definition (17) of $f$ as

$$
f(x)=\sum_{-\infty}^{-1} \frac{1}{|N|} p^{-1 / 2} \chi\left(p^{N-1} x\right) \Delta_{0}\left(p^{N} x\right) .
$$

We consider the sequence $x_{n}=p^{n}, n \in \mathbb{N}$. It is easy to see $x_{n} \rightarrow 0,(n \rightarrow \infty)$ in the $p$-adic norm $|\cdot|_{p}$. Moreover, $\Delta_{0}\left(p^{N} x_{n}\right)=\Delta_{0}\left(p^{N+n}\right)=0$ when $N+n \leq-1$. On the other hand, when $N+n \leq 1, p^{N-1} x_{n}$ become an integer $p$-adic number, and then $\chi\left(p^{N-1} x_{n}\right)=\chi\left(p^{N+n-1}\right)=1$. From the above relations and (20) we can get that

$$
\begin{aligned}
f\left(x_{n}\right) & =f\left(p^{n}\right) \\
& =p^{-1 / 2}\left(\frac{\chi\left(p^{-1}\right)}{n}+\sum_{N=-n+1}^{-1} \frac{1}{|N|}\right) \\
\text { as } n & \longrightarrow \infty .
\end{aligned}
$$

Therefore, $f(x)$ cannot be continuous at $x=0$.

\section{Pseudodifferential Equation}

In this section we will consider the pseudodifferential equation

$$
T^{\alpha} u+u=\delta_{x_{k}}, \quad u \in L_{2}\left(\mathbb{Q}_{p}\right), x_{k} \in \mathbb{Q}_{p},
$$

where $T^{\alpha}: L_{2}\left(\mathbb{Q}_{p}\right) \rightarrow S^{\prime}\left(\mathbb{Q}_{p}\right)$ is pseudodifferential operator in the distribution sense. 
Theorem 4. Equation (22) has a unique solution $u=u_{k} \in$ $L_{2}\left(\mathbb{Q}_{p}\right)$ for $\alpha>1 / 2$ and has no solutions belonging to $L_{2}\left(\mathbb{Q}_{p}\right)$ for $\alpha \leq 1 / 2$.

Proof. Let $\varphi \in D\left(T^{\alpha}\right)$. Similar to the proof of Theorem 2, we give the expansion of $\varphi$ using the uniformly convergent series with respect to the complex-conjugated $p$-adic wavelet basis $\left\{\overline{\psi_{N j I}}\right\}$. For $\left\{\overline{\psi_{N j I}}\right\}$ are continuous functions on $\mathbb{Q}_{p}$ we can write

$$
\varphi\left(x_{k}\right)=\sum_{N=-\infty}^{+\infty} \sum_{j=1}^{p-1} \sum_{I}\left(\varphi, \overline{\psi_{N j I}}\right) \overline{\psi_{N j I}}\left(x_{k}\right)
$$

for $x=x_{k}$.

$$
\begin{aligned}
\text { Consider } & \\
\overline{\psi_{N j I}}\left(x_{k}\right) & =p^{-N / 2} \overline{\chi\left(p^{N-1} j x_{k}\right)} \Delta_{0}\left(p^{N} x_{k}-z_{I}\right) \\
& =p^{-N / 2} \chi\left(-p^{N-1} j x_{k}\right) \Delta_{0}\left(p^{N} x_{k}-z_{I}\right) .
\end{aligned}
$$

It is easy to see $\overline{\psi_{N j I}}\left(x_{k}\right) \neq 0$ equals $\left|p^{N} x_{k}-z_{I}\right| \leq 1$. Here $I=z_{I}+B_{0} \in \mathbb{Q}_{p} / B_{0}$ and by the strong triangle inequality we obtain $\left|p^{N} x_{k}-z_{I}\right| \leq 1$ equals $z_{I}=\left\{p^{N} x_{k}\right\}$. Then, we obtain the conclusion that

$$
\overline{\psi_{N j I}}\left(x_{k}\right)= \begin{cases}0, & z_{I} \neq\left\{p^{N} x_{k}\right\}, \\ p^{-N / 2} \chi\left(-p^{N-1} j x_{k}\right), & z_{I}=\left\{p^{N} x_{k}\right\} .\end{cases}
$$

And then

$$
\begin{aligned}
\left\langle\delta_{x_{k}}, \varphi\right\rangle & =\varphi\left(x_{k}\right) \\
& =\sum_{N=-\infty}^{+\infty} \sum_{j=1}^{p-1} p^{-N / 2} \chi\left(-p^{N-1} j x_{k}\right)\left(\varphi, \overline{\psi_{N j\left\{p^{N} x_{k}\right\}}}\right) \\
& =\sum_{N=-\infty}^{+\infty} \sum_{j=1}^{p-1} p^{-N / 2} \chi\left(-p^{N-1} j x_{k}\right)\left\langle\psi_{N j\left\{p^{N} x_{k}\right\}}, \varphi\right\rangle .
\end{aligned}
$$

For $S\left(\mathbb{Q}_{p}\right) \subset D\left(T^{\alpha}\right)$, the equality (26) implies that

$$
\delta_{x_{k}}=\sum_{N=-\infty}^{+\infty} \sum_{j=1}^{p-1} p^{-N / 2} \chi\left(-p^{N-1} j x_{k}\right) \psi_{N j\left\{p^{N} x_{k}\right\}},
$$

in which series converges uniformly in $S^{\prime}\left(\mathbb{Q}_{p}\right)$.

We suppose that there is a function $u_{k} \in L_{2}\left(\mathbb{Q}_{p}\right)$ which can be represented as a convergent series in $L_{2}$ :

$$
u_{k}(x)=\sum_{N=-\infty}^{+\infty} \sum_{j=1}^{p-1} \sum_{I} c_{N j I} \psi_{N j I}(x) .
$$

Applying pseudodifferential operator $T^{\alpha}+I$ on the both sides, we obtain a series with (12)

$$
\begin{aligned}
T^{\alpha} u_{k}+ & u_{k} \\
= & \sum_{N=-\infty}^{0} \sum_{j=1}^{p-1} \sum_{I} c_{N j I}\left(1+p^{\alpha(1-N)}\right) \psi_{N j I} \\
& +\sum_{N=1}^{+\infty} \sum_{j=1}^{p-1} \sum_{I} 2 c_{N j I} \psi_{N j I},
\end{aligned}
$$

which converges in $S^{\prime}\left(\mathbb{Q}_{p}\right)$ for $T^{\alpha} S\left(\mathbb{Q}_{p}\right) \subset L_{2}\left(\mathbb{Q}_{p}\right)$. Comparing the two series (27) and (29), we obtain

$$
c_{N j\left\{p^{N} x_{k}\right\}}= \begin{cases}\frac{1}{2} p^{-N / 2} \chi\left(-p^{N-1} j x_{k}\right), & N \geq 1, \\ \left(p^{\alpha(1-N)}+1\right)^{-1} p^{-N / 2} \chi\left(-p^{N-1} j x_{k}\right), & N<1 .\end{cases}
$$

Thus,

$$
\begin{aligned}
u_{k}(x)=\sum_{N=1}^{+\infty} \sum_{j=1}^{p-1} \frac{1}{2} p^{-N / 2} & \\
& \times \chi\left(-p^{N-1} j x_{k}\right) \psi_{N j\left\{p^{N} x_{k}\right\}}(x) \\
& +\sum_{N=-\infty}^{0} \sum_{j=1}^{p-1}\left(p^{\alpha(1-N)}+1\right)^{-1} p^{-N / 2} \\
& \quad \times \chi\left(-p^{N-1} j x_{k}\right) \psi_{N j\left\{p^{N} x_{k}\right\}}(x) .
\end{aligned}
$$

Next, We will show that the series (31) belong to $L_{2}\left(\mathbb{Q}_{p}\right)$ for $\alpha>1 / 2$. For the general term of the first series we obatin

$$
\left|\frac{1}{2} p^{-N / 2} \chi\left(-p^{N-1} j x_{k}\right)\right|^{2} \leq \frac{1}{4} p^{-N}, \quad N \geq 1
$$

that means the first series converges in $L_{2}\left(\mathbb{Q}_{p}\right)$ for any $\alpha \in \mathbb{R}$.

We estimate the general term of the second series as follows:

$$
\begin{aligned}
& \left|\left(p^{\alpha(1-N)}+1\right)^{-1} p^{-N / 2} \chi\left(-p^{N-1} j x_{k}\right)\right|^{2} \\
& \quad \leq p^{-N}\left(p^{\alpha(1-N)}+1\right)^{-2} \leq p^{-2 \alpha} p^{-N(1-2 \alpha)}, \quad N<1 .
\end{aligned}
$$

It is easy to see the second series converges in $L_{2}\left(\mathbb{Q}_{p}\right)$ for $\alpha>$ $1 / 2$.

Thus $u_{k}$ is a unique solution of (31) for $\alpha>1 / 2$.

For $p^{\alpha(1-N)}+1 \leq 2 p^{\alpha(1-N)}$ with $N<1$, we estimate the general term of the second series as follows:

$$
\begin{gathered}
\left|\left(p^{\alpha(1-N)}+1\right)^{-1} p^{-N / 2} \chi\left(-p^{N-1} j x_{k}\right)\right|^{2} \\
\geq \frac{1}{4} p^{-2 \alpha} p^{-N(1-2 \alpha)}, \quad N<1
\end{gathered}
$$

that means the series diverges in $L_{2}\left(\mathbb{Q}_{p}\right)$ for $\alpha \leq 1 / 2$. Theorem 4 is proved.

Theorem 5. The solution $u_{k}$ of $(22)$ is continuous on $\mathbb{Q}_{p}$ for $\alpha>1$.

Proof. We will show that the series (31) converges uniformly on $\mathbb{Q}_{p}$ if $\alpha>1$ and converges uniformly on the ball not containing $x_{k}$ if $1 / 2<\alpha \leq 1$.

Indeed, the general term (31) of the first series does not exceed $(1 / 2) p^{-N}$ by (14) when $N \geq 1$. Thus, the first series converges uniformly with $N \geq 1$. 
When $N<1$ the general term (31) of the second series satisfies

$$
\left(p^{\alpha(1-N)}\right)^{-1} p^{-N} \leq p^{-\alpha} p^{-N(1-\alpha)} .
$$

The estimate we obtained above implies that for $\alpha>1$ the subseries of (31) converges uniformly with $N<1$. Hence, the series (31) converges uniformly for $\alpha>1$. Theorem 5 is proved.

Let $\alpha>1 / 2$ and fixed points $x_{1}, x_{2}, \ldots, x_{n} \in \mathbb{Q}_{p}$. Then, define $S p\left\{u_{k}\right\}_{1}^{n}$ as the linear span of solutions $u_{k}(k=1,2$, $\ldots, n)$ of $(22)$. Hence, we have the following.

Theorem 6. $S p\left\{u_{k}\right\}_{1}^{n} \cap D\left(T^{\alpha / 2}\right)=\{0\}$ for $1 / 2<\alpha \leq 1$ and $S p\left\{u_{k}\right\}_{1}^{n} \subset D\left(T^{\alpha / 2}\right)$ for $\alpha>1$.

Proof. The solution $u_{k}$ of (22) is obtained by (31). Considering the expansion (31) and semigroup property (c.f. [19])

$$
T^{\alpha_{1}} T^{\alpha_{2}}=T^{\alpha_{1}+\alpha_{2}}, \quad \alpha_{1}, \alpha_{2}>0
$$

of $T^{\alpha}$. Obviously, $u_{k} \in D\left(T^{\alpha / 2}\right)$ if and only if the following series converge in $L_{2}\left(\mathbb{Q}_{p}\right)$ :

$$
\begin{aligned}
& \sum_{N=1}^{+\infty} \sum_{j=1}^{p-1} \frac{1}{2} p^{-N / 2} \chi(\left.-p^{N-1} j x_{k}\right) \psi_{N j\left\{p^{N} x_{k}\right\}}(x) \\
&+\sum_{N=-\infty}^{0} \sum_{j=1}^{p-1}\left(p^{\alpha(1-N)}+1\right)^{-1} p^{-N / 2} \\
& \quad \times \chi\left(-p^{N-1} j x_{k}\right) p^{(\alpha / 2)(1-N)} \psi_{N j\left\{p^{N} x_{k}\right\}}(x) .
\end{aligned}
$$

For the general term of the first series, we have the estimate as follows:

$$
\left|\frac{1}{2} p^{-N / 2} \chi\left(-p^{N-1} j x_{k}\right)\right|^{2} \leq \frac{1}{4} p^{-N}, \quad N \geq 1
$$

that means it converges in $L_{2}\left(\mathbb{Q}_{p}\right)$ for any $\alpha \in \mathbb{R}$. have

Similarly, for the general term of the second series, we

$$
\begin{aligned}
& \left|\left(p^{\alpha(1-N)}+1\right)^{-1} p^{-N / 2} \chi\left(-p^{N-1} j x_{k}\right) p^{(\alpha / 2)(1-N)}\right|^{2} \\
& \quad \leq C p^{(\alpha-1) N}, \quad N<1 .
\end{aligned}
$$

Hence the series converges when $\alpha>1$. Then $S p\left\{u_{k}\right\}_{1}^{n} \subset$ $D\left(T^{\alpha / 2}\right)$ if $\alpha>1$.

For $p^{\alpha(1-N)}+1 \leq 2 p^{\alpha(1-N)}$ with $N<1$, the general term of the second series can be estimated below

$$
\begin{gathered}
\left|\left(p^{\alpha(1-N)}+1\right)^{-1} p^{-N / 2} \chi\left(-p^{N-1} j x_{k}\right) p^{(\alpha / 2)(1-N)}\right|^{2} \\
\geq \frac{1}{4} p^{-\alpha} p^{-N(1-\alpha)}, \quad N<1
\end{gathered}
$$

that means it diverges in $L_{2}\left(\mathbb{Q}_{p}\right)$ for $\alpha \leq 1$.
Hence $u_{k} \notin D\left(T^{\alpha / 2}\right)$. Since the estimate (40) does not depend on the choice of $u_{k}$. And considering the functions $\left\{\psi_{N j\left\{p^{N} x_{k}\right\}}(x)\right\}(N<1)$ of the basis $\left\{\psi_{N j I}(x)\right\}$ in the $u_{k}(k=$ $1,2, \ldots, n)$ of (31) are different for any small negative $N$, we obtain that $S p\left\{u_{k}\right\}_{1}^{n} \cap D\left(T^{\alpha / 2}\right)=\{0\}$ if $1 / 2<\alpha \leq 1$. Theorem 6 is proved.

\section{Conclusions}

In this paper, solutions of pseudodifferential equations with the type $T^{\alpha} u+u=\delta_{x_{k}}$ over $p$-adic field $\mathbb{Q}_{p}$ are considered. First, we give the condition for the continuity of arbitrary function in the domain of definition $D\left(T^{\alpha}\right)$ with the index $\alpha>1 / 2$ and give the example to show noncontinuity when $\alpha \leq 1 / 2$. Then, we obtain the existence of solutions to the pseudodifferential equations. The results show that the equation has a unique solution belonging to $L_{2}\left(\mathbb{Q}_{p}\right)$ for $\alpha>$ $1 / 2$ and has no solutions for $\alpha \leq 1 / 2$. Furthermore, we show the continuity of solutions when $\alpha>1$. Finally, the embedding from the linear span of solutions to the domain of definition $D\left(T^{\alpha}\right)$ is obtained.

\section{Acknowledgments}

The author would like to thank the Academic Editor Guocheng Wu and all anonymous reviewers for their kind support which helped the author to improve the paper considerably. This work was supported by the National Natural Science Foundation of China under NSFS nos. 11071109, 11001119 and the Priority Academic Program Development of Jiangsu Higher Education Institutions (PAPD).

\section{References}

[1] S. Albeverio and W. Karwoski, "Diffusion in p-adic numbers," in Gaussian Random Fields, K. Ito and H. Hida, Eds., pp. 86-99, World Scientific, Singapore, 1991.

[2] S. Albeverio and W. Karwowski, "A random walk on p-adics: the generator and its spectrum," Stochastic Processes and their Applications, vol. 53, no. 1, pp. 1-22, 1994.

[3] S. Albeverio and P. Kurasov, "Singular perturbations of differential operators," in Solvable Schrödinger Type Operators, vol. 271 of London Mathematical Society Lecture Note Series, Cambridge University Press, Cambridge, UK, 2000.

[4] S. Albeverio, A. Yu. Khrennikov, and V. M. Shelkovich, "Harmonic analysis in the $p$-adic Lizorkin spaces: fractional operators, pseudo-differential equations, $p$-adic wavelets, Tauberian theorems," The Journal of Fourier Analysis and Applications, vol. 12, no. 4, pp. 393-425, 2006.

[5] S. Albeverio and S. Kuzhel, "One dimensional Schrödinger operators with $p$-symmertric zero-range potentials," Journal of Physics A, vol. 38, pp. 4975-4988, 2005.

[6] V. A. Avetisov, A. H. Bikulov, S. V. Kozyrev, and V. A. Osipov, “padic models of ultrametric diffusion constrained by hierarchical energy landscapes," Journal of Physics A, vol. 35, no. 2, pp. 177$189,2002$.

[7] V. A. Avetisov, A. Kh. Bikulov, and V. Al. Osipov, "p-adic description of characteristic relaxation in complex systems," Journal of Physics A, vol. 36, no. 15, pp. 4239-4246, 2003. 
[8] A. Khrennikov, p-Adic Valued Distributions in Mathematical Physics, vol. 309, Kluwer Academic Publishers, Dordrecht, The Netherlands, 1994.

[9] A. Khrennikov, Non-Archimedean Analysis: Quantum Paradoxes, Dynamical Systems and Biological Models, vol. 427, Kluwer Academic Publishers, Dordrecht, The Netherlands, 1997.

[10] A. N. Kochubei, "Parabolic equations over the field of $p$-adic numbers," Mathematics of the USSR-Izvestiya, vol. 39, pp. 1263$1280,1992$.

[11] A. N. Kochubei, Pseudo-Differential Equations and Stochastics over Non-Archimedean Fields, Marcel Dekker, New York, NY, USA, 2001.

[12] V. S. Varadarajan, "Path integrals for a class of $p$-adic Schrödinger equations," Letters in Mathematical Physics, vol. 39, no. 2, pp. 97-106, 1997.

[13] V. S. Vladimirov, I. V. Volovich, and E. I. Zelenov, p-Adic Analysis and Mathematical Physics, World Scientific, Singapore, 1994.

[14] S. Kuzhel and S. Torba, " $p$-adic fractional differentiation operator with point interactions," Methods of Functional Analysis and Topology, vol. 13, no. 2, pp. 169-180, 2007.

[15] S. Albeverio, S. Kuzhel, and S. Torba, " $p$-adic Schrödingertype operator with point interactions," Journal of Mathematical Analysis and Applications, vol. 338, no. 2, pp. 1267-1281, 2008.

[16] S. Hassi and S. Kuzhel, "On symmetries in the theory of finite rank singular perturbations," Journal of Functional Analysis, vol. 256, no. 3, pp. 777-809, 2009.

[17] W. Y. Su, "Pseudo-differential operators and derivatives on locally compact Vilenkin groups," Science in China A, vol. 35, no. 7, pp. 826-836, 1992.

[18] M. H. Taibleson, Fourier Analysis on Local Fields, Princeton University Press, Princeton, NJ, USA, 1975.

[19] H. Qiu and W. Y. Su, "Pseudo-differential operators over $p$-adic fields," Science in China A, vol. 41, no. 4, pp. 323-336, 2011. 


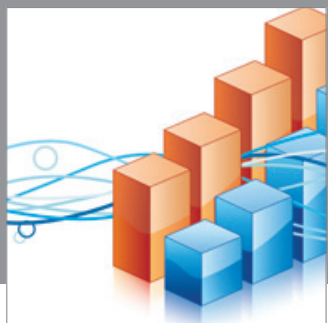

Advances in

Operations Research

mansans

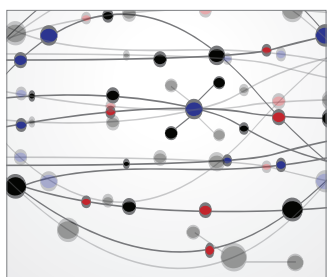

The Scientific World Journal
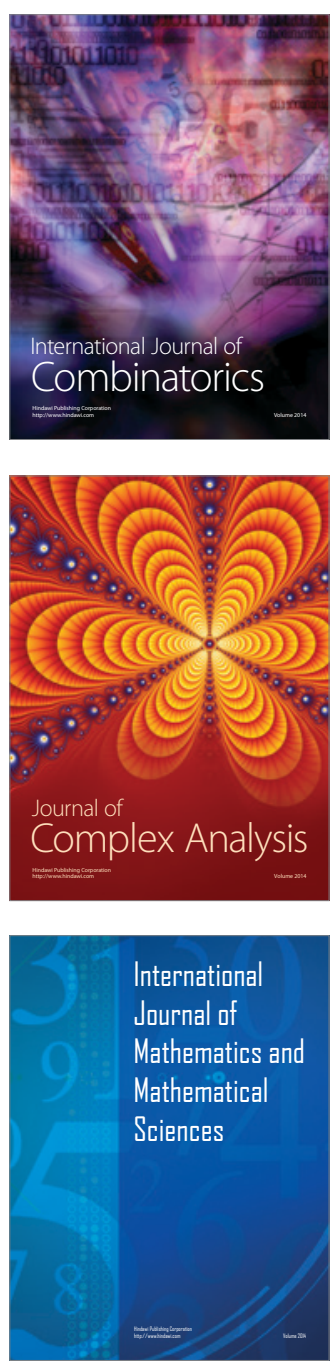
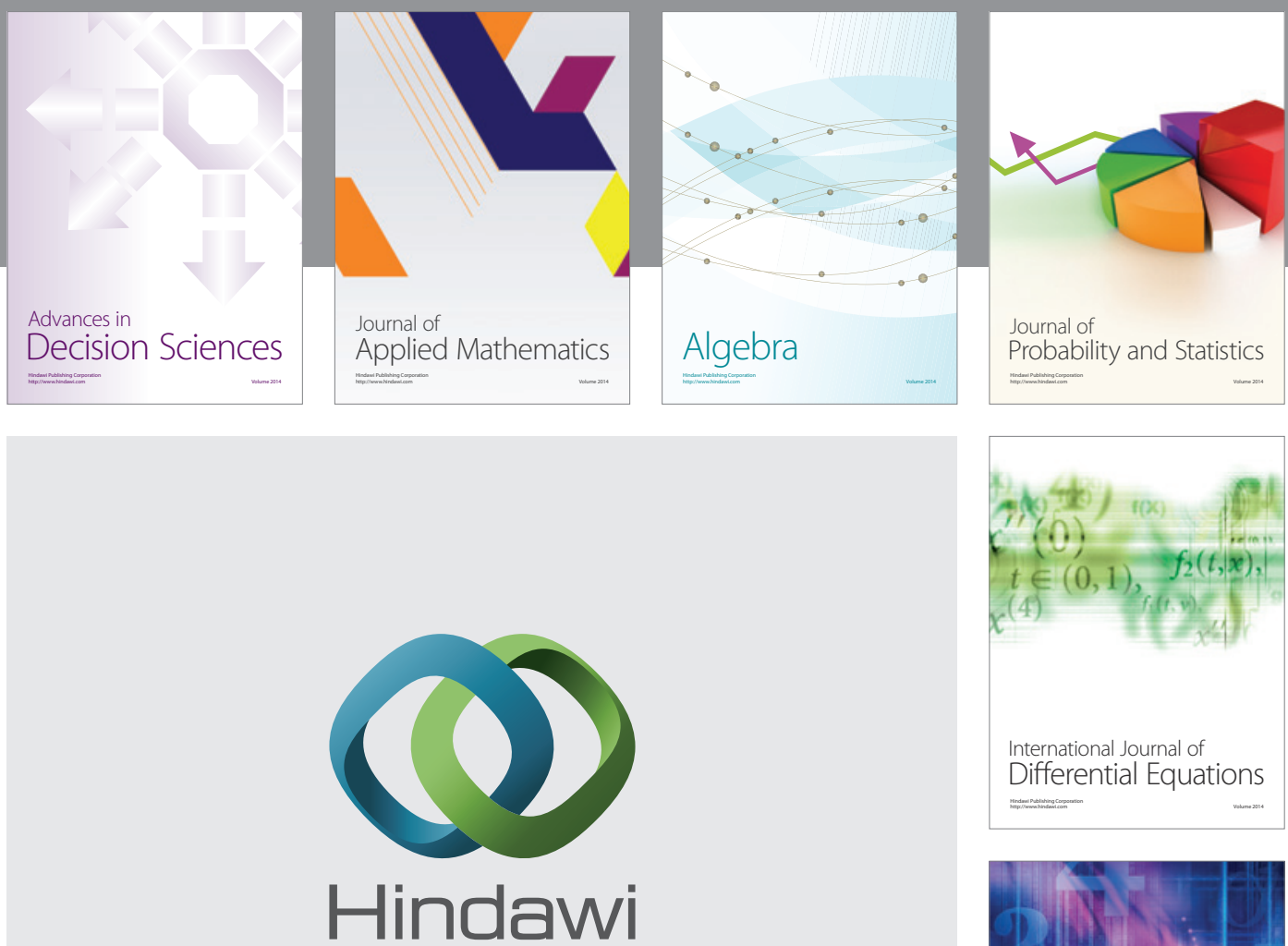

Submit your manuscripts at http://www.hindawi.com
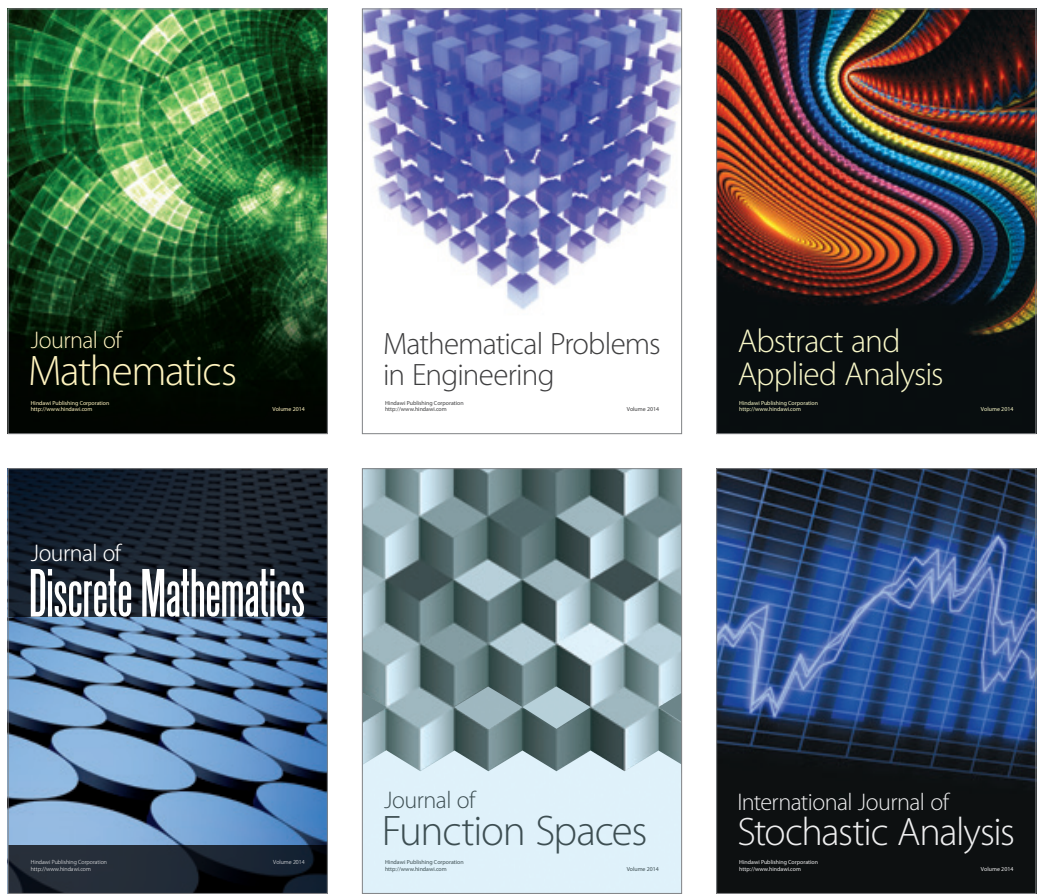

Journal of

Function Spaces

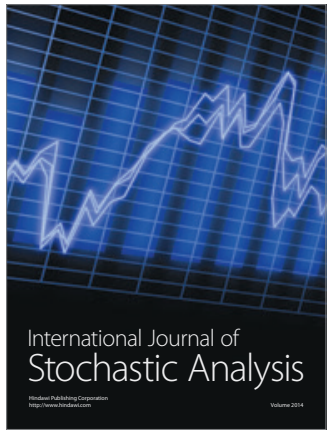

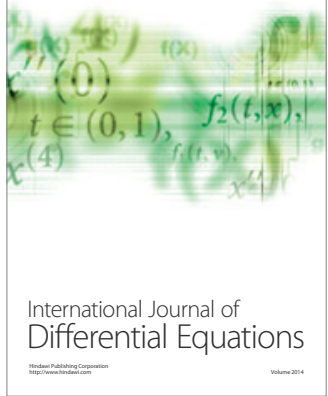
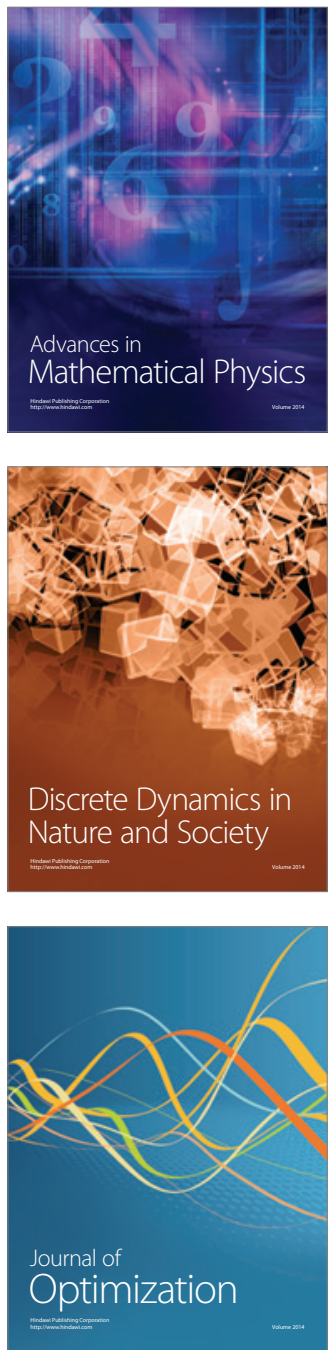